\title{
FIXED LINK MARGINS OUTPERFORM POWER CONTROL IN ENERGY-LIMITED WIRELESS SENSOR NETWORKS
}

\author{
Mathias Johansson, Erik Björnemo and Anders Ahlén \\ Signals and Systems, Uppsala University, Box 534, 75121 Uppsala, Sweden. \\ E-mail:\{mj, eb, aa\}@angstrom.uu.se
}

\begin{abstract}
We investigate whether adapting the transmit power optimally to the time-varying channel compares favorably, in terms of total energy consumption, to using a fixed link margin in wireless networks over short transmission distances. Over short distances, the circuit energy consumption dominates the transmission energy. For that reason, feeding back channel state information - a requirement for power control - may not be a power efficient strategy. We investigate both slow and fast power control and conclude, somewhat surprisingly, that using a fixed margin is typically more power-efficient than using power control.
\end{abstract}

Index Terms- Sensor networks, energy efficiency, power control, feedback, fading

\section{INTRODUCTION}

Wireless sensor networks (WSN) are fundamentally different from cellular networks. Whereas cellular networks are characterized by high data rates and large transmission distances, wireless sensor nodes operate at low bit-rates and short distances. Due to the large distances and high data rate requirements, the energy expenditure in a cellular unit is dominated by transmission energy. In a short-range low bit-rate WSN, the situation is radically different. Here, transmission energy is small compared to the energy required by the radio transceiver circuitry, and consequently traditional results on energyefficient designs must be reexamined. In existing work on cellular systems and other high-rate long-range applications, the circuit energy is neglected and only the transmit energy is optimized. In WSN, the inefficiencies of the circuitry must be kept in mind at all times, and more often than not lead to opposite conclusions than when focus is exclusively on transmission energy. In a previous study [1] we re-addressed the question of whether collaborative space-time block coding (STBC) has an advantage over simple point-to-point communication. Although previous results (see for example [2], [3]) had indicated that STBC could yield dramatic energy savings, we found the opposite to be true in the great majority of cases by varying critical parameters over realistic ranges. Only with very large - if not excessive - link margins and pure Rayleigh fading would it be possible to recommend collaborative STBC.

Here, our focus is on the link margin itself and the option to adapt transmission power. To ensure a relatively constant bit-error rate over a time-varying fading channel, transmit power must either be set high enough so that even when channel attenuation is greater than on average, the bit-error rate (BER) will stay on an acceptable level, or the power level must be adapted to the current channel conditions. In order to adapt the transmit power, the power amplifier (PA) on the transmitting end must have a sufficient dynamic range and allow for continuous adjustments, and, more significantly, channel feedback must be available so that the correct transmit power level is chosen. This requires that the receiver measures (and predicts) the channel attenuation and transmits some quantized channel state information (CSI) back to the original transmitter which then adjusts the transmit power accordingly. Will the additional energy expenditure outbalance the transmit energy reduction? Obviously, the answer depends on a realistic energy consumption model, a matter which we turn to in the next section. Our analysis necessarily requires assumptions about circuit energy consumption, but we give results that reflect what would happen if advances in energy-efficient circuitry are made in the future. Our basic scenario uses parameter values that are representative of good circuit designs today.

It is worth noting that the term power control is also used on the network level, where transmit power is adjusted to establish a certain network topology, that is, to ensure connectivity between nodes of some distance. In cellular systems, power control is also used for interference mitigation, see for example [4]. Our topic is different: we wish to see whether adapting the transmit power to the timevarying channel variations for a given BER requirement is more or less energy efficient than using a fixed transmit power level.

\section{THE CHANNEL MODEL AND THE ENERGY CONSUMPTION MODEL}

We divide the channel attenuation into two components: one slowly varying, and one fast varying. The slowly varying part is defined as the path loss due to distance and shadowing from large objects, whereas the fast component is due to the movements of small objects. We assume log-normal statistics for the slowly varying part, and the fast component is modelled by a Nakagami- $m$ probability distribution.

We investigate two different ways of determining the transmit power. The simplest scheme is to simply fix the transmit power to some value that on average achieves a given bit error requirement. Thus, in order to cope with the unknown channel fluctuations a link safety margin is introduced. This implies that energy is sometimes wasted by transmitting at an unnecessarily high power level. One might suspect that if the required link safety margin is very high due to large fluctuations over large time scales, power could be saved by implementing a CSI feedback scheme so that the transmit power could be adjusted to the instantaneous channel conditions. Then, transmit power would never be wasted. This is our second method, which we refer to as power control. We will distinguish between slow power control, which only adapts to the slowly varying channel component, and fast power control, which also adapts to the fast variations. 


\subsection{Transmit energy consumption}

The required transmit energy (to be precise: input energy to the PA) per bit is given by

$$
E=\frac{1}{\gamma} \frac{M L N_{f}}{G} \bar{E}
$$

with $\gamma$ the efficiency of the PA, $M$ the loss due to slow fading, $L$ the path loss due to distance-related attenuation, $N_{f}$ the receiver noise figure, $G$ the combined antenna gain at the transmitter and the receiver, and $\bar{E}$ the required received energy per bit at the receiver. The required energy at the receiver is determined so that a certain BER $B$ can be attained on average, assuming that the statistics of the fast fading is accurately modelled by a distribution $f_{\mathcal{F}}(B)$, where $\mathcal{F}$ indexes the fast fading characteristics. In computing BER values we assume that coherently detected BPSK is employed. The BER for a channel corrupted only by additive white Gaussian noise is then

$$
B=Q\left(\sqrt{\frac{2 \bar{E}}{N_{0}}}\right),
$$

where $N_{0}$ is the noise power spectral density. We will then average this expression over the relevant fading distribution (Rayleigh, Nakagami- $m$, Log-normal) for the different cases we investigate below.

It can be noted that although interference is not explicitly mentioned in the model above, it is possible to interpret slowly varying interference as part of the path loss $L$, and fast varying interference as one of the parts that determine $f_{\mathcal{F}}(B)$.

\subsection{Circuit energy consumption}

On short transmission distances, circuit energy consumption is a major part of the total energy expenditure. This includes energy consumed by DAC:s, mixers, frequency synthesizers and low-noise amplifiers. For the transmission and reception of one bit, respectively, $E_{c t}$ and $E_{c r}$ Joules are required. These terms are constant with respect to transmit power and need not be separated into individual contributions from each RF component. We neglect any costs associated with computations and signal processing for carrying out the power control scheme.

\section{SLOW POWER CONTROL}

As stated above, we have chosen to make a distinction between fast channel variations, as captured by $f_{\mathcal{F}}(B)$, and slow variations, embodied in the loss $M$. Slow power control refers to a scheme where $M$ (and the constant path loss $L$ ) is tracked at the receiver and fed back to the transmitter so that no extra margin is needed. In this case, $\bar{E}$ is fixed for all transmissions. Given that we have sufficiently accurate knowledge of the fast fading statistics, we can determine an adequate $\bar{E}$ given a BER requirement.

Assume that $l$ payload bits per second are to be transmitted between two nodes in both directions and that slow power control is used at both ends both for payload communication and CSI communication. This simplifies the analysis somewhat. Alternatively, we could assume perfectly reciprocal channels and interference and then study only one-way communication. The channel may very well be reciprocal but the interference is typically not, and therefore we prefer to keep the analysis like this. We will however also investigate a scenario where the receiving node's energy consumption can be neglected in the next subsection.
Table 1. Summary of parameter values.

\begin{tabular}{|c|c|c|c|}
\hline Parameter & Value & Parameter & Value \\
\hline$G$ & $5 \mathrm{dBi}$ & $N_{f}$ & $10 \mathrm{~dB}$ \\
$\gamma$ & 0.35 & $N_{0}$ & $-204 \mathrm{dBJ}$ \\
\hline
\end{tabular}

We here make a simplified notation of the required transmit energy (1)

$$
E_{i}=K_{i} \bar{E}
$$

where power control requires that $K_{i}$ be estimated at the receiver and communicated back to the transmitter, and $i$ is either 1 or 2 for the respective directions. This CSI communication requires $n$ bits per second in either direction. We assume that $K_{i}$ is estimated and communicated without error. In computing $K_{i}$ we assume that $M=0 \mathrm{~dB}$ on average and use the fixed parameter values in Table 1 . Thus the only variable part in $K_{i}$ is the path loss $L$. Nonetheless, for compactness we ignore writing out that $K_{i}$ is in fact a function of $L$.

The total energy consumption for transmitting $n+l$ bits in both directions thus becomes

$$
E_{t o t, C S I}=\left(\left(K_{1}+K_{2}\right) \bar{E}+2 E_{c t}+2 E_{c r}\right)(l+n) .
$$

We compare this to the energy consumption of using a fixed link safety margin instead of relying on CSI feedback. Assuming that the extra link margin has to be a factor $X$ above the optimal value $K_{i}$ on average, we have the energy consumption

$$
E_{t o t, n o C S I}=\left(\left(K_{1} X+K_{2} X\right) \bar{E}+2 E_{c t}+2 E_{c r}\right) l
$$

Comparing the two cases (4) and (5), and rearranging the terms, we find that slow power control requires less energy than using a fixed margin if

$$
\frac{l+n}{l}+\frac{n}{l} \frac{2\left(E_{c t}+E_{c r}\right)}{\left(K_{1}+K_{2}\right) \bar{E}}<X
$$

The first term on the left hand side is approximately 1 , so that only if the ratio of the transmission energy per bit and the circuit energy per bit is of roughly the same order of magnitude as the ratio of the amount of CSI to the amount of payload data is slow power control more energy efficient. For instance, if we have to use CSI on the order of $1 \%$ of the payload data per second, the optimal transmission energy per bit also has to be at least on the order of $1 \%$ of the circuit energy per bit. However, at short distances (how short depends on the carrier frequency and the physical environment) the transmission energy is typically several orders of magnitude smaller than the circuit energy. For example, with Rayleigh fading and $B=10^{-3}$, we have $\bar{E}=9.9 \cdot 10^{-19} \mathrm{~J}$. Together with a path loss of $80 \mathrm{~dB}$, giving $K_{i} \approx 90 \mathrm{~dB}$, the total transmit energy becomes merely $0.9 \mathrm{~nJ}$. In Figure 1, the exact threshold margin is plotted versus the path loss using the parameter values listed in Table 1 under the assumption of Rayleigh fading. It can be seen that the path loss must be substantial, implying long transmission distances, for superiority of slow power control.

For instance, assuming free-space propagation and a center frequency of $868 \mathrm{Mhz}$, the distance has to be roughly $300 \mathrm{~m}$ for the path loss to exceed $80 \mathrm{~dB}$. At $2.5 \mathrm{GHz}$, the same path loss still requires a distance of $100 \mathrm{~m}$. At $80 \mathrm{~dB}$ path loss, the required link margin $X$ for the non-adaptive case must be higher than $23 \mathrm{~dB}$ if slow power control is to be more energy-efficient when $n / l=0.01$ (that is, $1 \%$ CSI), $B=10^{-3}$, and $E_{c t}=E_{c r}=10 \mu J$. A $10 \mathrm{~dB}$ reduction of 


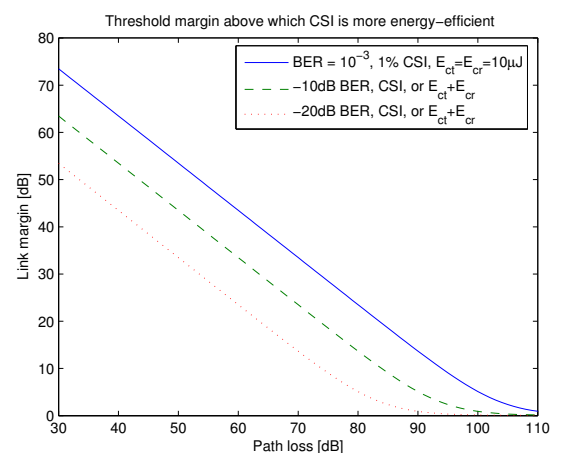

Fig. 1. Threshold link margin $X$ as a function of the path loss $L$ under the assumption of Rayleigh fading. Above the threshold, slow power control is more energy-efficient than the fixed margin. A reduction of a factor 10 of either the amount of CSI, the BER requirement, or the circuit energy $E_{c t}+E_{c r}$ all leads to a similar factor 10 reduction of the threshold (in the low path loss region).

Table 2. The required additional link margin $X$ required in order to keep the BER at a desired level as a function of the standard deviation $\sigma_{s}$ of the log-normal slow fading.

\begin{tabular}{|c|c||c|}
\hline BER & $\sigma_{s}$ & Required margin \\
\hline $10^{-3}$ & $10 \mathrm{~dB}$ & $1.8 \mathrm{~dB}$ \\
& $12 \mathrm{~dB}$ & $7.4 \mathrm{~dB}$ \\
\hline $10^{-4}$ & $10 \mathrm{~dB}$ & $0 \mathrm{~dB}$ \\
& $12 \mathrm{~dB}$ & $5.56 \mathrm{~dB}$ \\
\hline $10^{-5}$ & $10 \mathrm{~dB}$ & $0 \mathrm{~dB}$ \\
& $12 \mathrm{~dB}$ & $2.58 \mathrm{~dB}$ \\
\hline
\end{tabular}

circuit energy consumption, would still require a link margin higher than $13 \mathrm{~dB}$ for slow power control to be preferable.

Let us now make a rough estimation of a reasonable link margin in practice. In [5], results from an extensive measurement campaign (involving 95 macro-cells across the United States) were reported for $1.9 \mathrm{GHz}$. Among other findings, log-normal shadow fading statistics were collected and statistics for the standard deviation showed a variability between $5-12 \mathrm{~dB}$. No standard deviation larger than 12 $\mathrm{dB}$ was ever observed, and the great majority of cases resided in a range between $6-9 \mathrm{~dB}$. To find out what a reasonable link margin would be in practice, we do the following calculation. Treating the fast fading and the slow fading as independent, we can attain the desired target BER by adjusting $\bar{E}$ for half the required BER, and then setting the link margin $X$ so that the slow fading incurs a similar BER. The mean SNR in the log-normal model is given by $\bar{E} X / N_{0}$ and averaging the BER for coherently detected BPSK in an additive white Gaussian noise-channel over the log-normal fading statistics with varying standard deviation $\sigma_{s}$ we can see how large $X$ should be to avoid exceeding the BER requirement. The results, obtained by numerical integration, are given in Table 2 for three different BER requirements. It can be noted that the mean signal level required for combatting the Rayleigh fading is in most cases also sufficiently large to ensure that the slow fading does not cause a BER in excess of the requirement. For the $\sigma_{s}=12 \mathrm{~dB}$ case however, a margin is needed. The margin is however small compared to the threshold margins according to Figure 1.

It can be noted that when we instead consider a more general

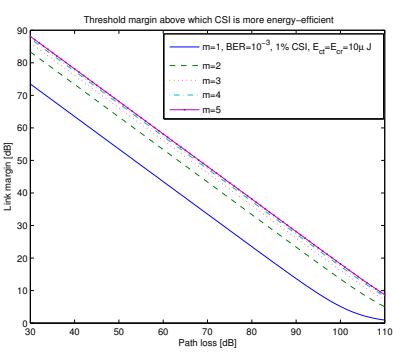

(a)

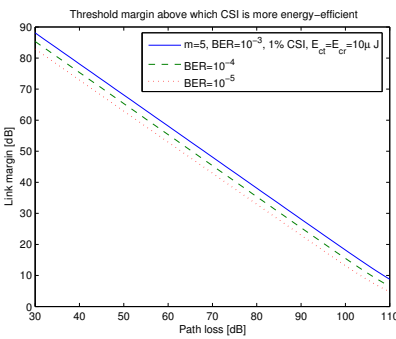

(b)
Fig. 2. (a) Threshold margin $X$ as a function of the fading figure $m$ in the Nakagami- $m$ fading model. The Rayleigh case corresponds to $m=1$. (b) Threshold margin $X$ as a function of the path loss $L$ under different BER requirements assuming a Nakagami- $m$ distribution with $m=5$.

Nakagami- $m$ fast fading model and let the fading figure $m$ increase ( $m=1$ corresponds to Rayleigh fading) thereby assuming a less fluctuating channel, the required transmit energy is lowered. Thus, the threshold margin increases further when the fast fading becomes more static. This is illustrated in Figure 2(a), where the impact of the fading figure is shown. Figure 2(b) plots the threshold dependence on BER for a fading figure $m=5$, where we see that tightening the BER requirement does not have as much impact as when $m=1$. A reduction of the circuit energy or the CSI would however still follow the same linear relationship as for $m=1$, that is, a factor 10 increase or decrease leads to $10 \mathrm{~dB}$ higher or lower thresholds respectively in the low path loss region.

The inevitable conclusion is that for networks operating on transmission distances up to a few hundred meters, energy efficient communication requires that slow power control be avoided.

Finally, we should also keep in mind that the sensitivity of typical transceiver circuits for sensor nodes do not allow transmission over larger path losses than around $90-110 \mathrm{~dB}$, which means that in cases when slow power control could be useful, the nodes may not be able to operate anyway.

\subsection{Node-to-hub Communication}

In the examples above, we have aimed at optimizing the energy consumption in both nodes. This is generally the design objective when both nodes run on limited battery supplies. In networks where a node communicates with a central hub, the hub may however have a continuous power supply. In that case, we might only want to consider the energy consumption in the transmitting node. When using power control, the energy consumption in the node is

$$
\left(K_{1} \bar{E}+E_{c t}\right) l
$$

for communicating payload data, and for the reception of CSI:

$$
E_{c r} n \text {. }
$$

Using instead a fixed margin, the energy consumption in the node would be

$$
\left(K_{1} X \bar{E}+E_{c t}\right) l \text {. }
$$

Comparing the two cases, we find that slow power control is more energy efficient than a fixed margin if

$$
1+\frac{n}{l} \frac{E_{c r}}{K_{1} \bar{E}}<X
$$




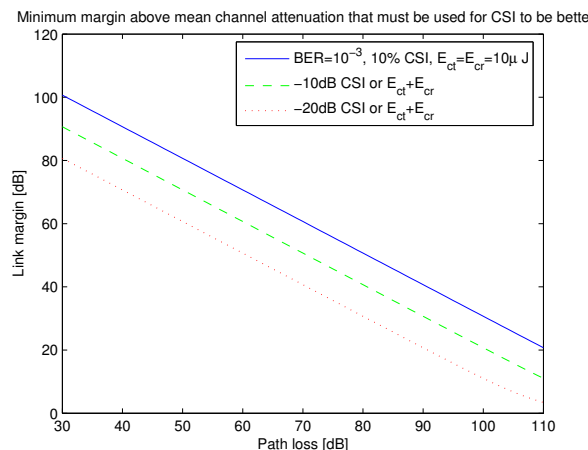

Fig. 3. Total threshold margin $X$ as a function of the path loss $L$ under the assumption of Rayleigh fading when comparing fast power control with a fixed link margin.

The threshold no longer depends on $E_{c t}$ (but of course it still affects the total energy expenditure). Comparing (10) to the previous case (6) we see that when $K_{1}=K_{2}$ and $E_{c t}=E_{c r}$ the only difference is that the threshold $X$ is reduced a factor 2 (assuming that $\frac{l+n}{l} \approx 1$ ). In conclusion, as expected, we find that taking only the energy consumption in the transmitting node into account the threshold simply reduces $3 \mathrm{~dB}$ as compared to the results in Figure 1.

\section{FAST POWER CONTROL}

Fast power control refers to the case when the CSI rate is sufficiently high to allow also the fast fading component to be tracked. In this case, equations (1)-(6) still apply with a slight reinterpretation: $\bar{E}$ is now set for a channel without any fading but corrupted by Gaussian receiver noise. For the fixed margin scenario, the fading margin $X$ now accounts for both slow and fast fading. The total threshold margin above which fast power control is more energy-efficient than using a fixed margin is plotted in Figure 3 using similar parameters as previously except that the CSI is increased to $10 \%$ of the payload data, and assuming Rayleigh fading.

For ease of interpretation, we split the total required margin $X$ into one component that handles the fast fading and one that accounts for the slow fading. At a required total BER of $10^{-3}$ the fixed margin for the fast Rayleigh component is roughly $17 \mathrm{~dB}$ using the standard BER formulas for coherently detected BPSK. We can see in Figure 3 that if we would then add a $10 \mathrm{~dB}$ margin for the slow component (larger than what is indicated in Table 2), the path loss required for break-even between the two techniques is a hefty $103.5 \mathrm{~dB}$. At 868 $\mathrm{MHz}$ and free-space propagation this corresponds to a distance of more than $4 \mathrm{~km}$, and at $2.5 \mathrm{GHz} 1.4 \mathrm{~km}$. Of course, we must keep in mind that this assumes that the CSI required is $10 \%$ of the payload data (10 times more than our baseline case for slow power control) and the circuit energies $E_{c t}=E_{c t}=10 \mu \mathrm{J}$. Even so, a $10 \mathrm{~dB}$ reduction of any of these two quantities transfers into a respectable $93.5 \mathrm{~dB}$ path-loss for break-even $(1.3 \mathrm{~km}$ at $868 \mathrm{MHz}$ and $450 \mathrm{~m}$ at $2.4 \mathrm{GHz})$.

\section{EFFICIENCY REDUCTIONS DUE TO POWER CONTROL}

Above we have assumed a constant efficiency of the power amplifier. In reality however, the efficiency reaches its maximum near the maximum output power. The efficiency drops with output power back-off, which further reduces any potential benefits of power control. For an ideal class B stage, the efficiency drops by a factor $p$ if the RF input level is reduced by a factor $p$ (amplitude, not power) (see [6], Ch. 8). For instance, a $3 \mathrm{~dB}$ reduction of drive power leads to an efficiency reduction from the maximum $78.5 \%$ to $55.5 \%$. The efficiency reduction is typically similar for a class $\mathrm{AB}$ amplifier whereas it is even worse for a class A design. An ideal class A amplifier may actually go from a maximum efficiency at nearly $50 \%$ to a mere $5 \%$ when output power is backed off $10 \mathrm{~dB}$. In order to alleviate these problems and maintain a more constant efficiency one can for instance use bias adaptation. In practice, this has to be balanced against increased costs of the transceiver. It is worth emphasizing that in our analysis above we have assumed constant efficiency regardless of back-off. In the high-path loss scenarios where it might possibly be appealing to use power control, it is important to make a refined analysis taking these effects into account.

\section{CONCLUSIONS}

Our analysis shows, perhaps surprisingly, that in typical WSN scenarios power control wastes energy as compared to using a fixed link margin. With high path losses, very efficient circuits (e.g., based on $M$-FSK modulation), or very low CSI feedback rates, power control can however be an interesting technique for energy reduction and increased robustness. For instance, the lowest curve in Figure 1 (that is, CSI rates of $0.01 \%$ of payload data) indicates that an initial power calibration upon deployment may be reasonable. Coupled with a possibility of an on-demand reset of the network with a renewed calibration the robustness of the network may also increase.

In light of the relation between transmission energy and circuit energy at short distances, on a more general level our recommendation is to spend little effort on advanced transmission techniques in WSN contexts. Any extra transmission incurs a cost that outweighs almost any reduction in transmission energy. As a final reminder, the results reported here have important implications also for the feasibility of ARQ, coding and rate optimization.

\section{REFERENCES}

[1] E. Björnemo, M. Johansson and A. Ahlén, "SISO outperforms cooperative MIMO in energy-limited wireless sensor networks", Submitted, 2006.

[2] S. Cui, A. J. Goldsmith and A. Bahai, "Energy-efficiency of MIMO and cooperative MIMO techniques in sensor networks", IEEE J. Sel. Areas Commun., vol. 22, no. 6, pp. 1089-1098, Aug. 2004.

[3] S. K. Jayaweera, "Energy analysis of MIMO techniques in wireless sensor networks", CISS 04, Princeton, New Jersey, Mar. 2004.

[4] J. Zander, "Performance of optimum transmitter power control in cellular radiosystems ", IEEE Transactions on Vehicular Technology., vol. 41, no. 1, pp. 57-62, Feb. 1992.

[5] V. Erceg, L. J. Greenstein, S. Y. Tjandra, S. R. Parkoff, A. Gupta, B. Kulic, A. A. Julius and R. Bianchi, "An empirically based path loss model for wireless channels in suburban environments", IEEE J. Sel. Areas Commun., vol. 17, no. 7, pp. 12051211, July 1999.

[6] Steve C. Cripps, RF Power Amplifiers for Wireless Communications, Artech House, 1999. 